

\title{
Isobaric beam purification for high precision Penning trap mass spectrometry of radioactive isotope beams with SWIFT
}

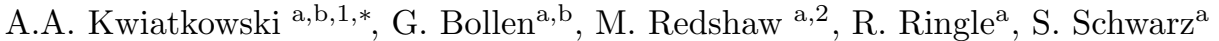 \\ ${ }^{a}$ National Superconducting Cyclotron Laboratory, Michigan State University, East Lansing, MI 48824, USA \\ ${ }^{b}$ Physics and Astronomy Department, Michigan State University, East Lansing, MI 48824, USA
}

\begin{abstract}
The Stored Waveform Inverse Fourier Transform (SWIFT) technique offers general and versatile broadband, mass-selective excitation schemes. The method has hitherto been unharnessed for high precision Penning trap mass spectrometry, in which the atomic mass is determined, and is of particular importance at radioactive isotope beam facilities. Herein, we present the first implementation of SWIFT to clean isobaric contaminants at such a spectrometer. The isobaric separation of $\mathrm{CO}^{+}$and $\mathrm{N}_{2}^{+}$as well as a high-accuracy mass measurement with SWIFT cleaning were demonstrated at the Low Energy Beam and Ion Trap (LEBIT) facility; resolving powers of $4 \times 10^{4}(\Delta \nu=300 \mathrm{~Hz})$ and $2 \times 10^{5}(\Delta \nu=60 \mathrm{~Hz})$ were achieved for ${ }^{23} \mathrm{Na}^{+}$for cleaning times of 20 and $100 \mathrm{~ms}$ respectively. These measurements demonstrate the viability of SWIFT for fast, efficient, and universal beam purification at high-precision Penning trap mass spectrometers.
\end{abstract}

Keywords: Penning trap, mass spectrometry, beam purification, atomic mass, radioactive isotope beams

\section{Introduction}

Knowledge of masses provides invaluable insight into a range of disciplines, from physics beyond the Standard Model to metrology [1]. At the forefront for precision mass measurements are those performed in a Penning trap mass spectrometer. The technique relies on measuring the cyclotron frequency, $2 \pi \nu_{c}=q / m \cdot B$, where $q / m$ is the chargeto-mass ratio and $B$ the magnetic field strength. $3 \mathrm{D}$ confinement is achieved by a superposition of a magnetic and electrostatic field, resulting in an axial oscillation, a magnetron orbit, and a reduced cyclotron eigenmotion instead of pure cyclotron motion [2]. The cyclotron frequency can be determined from coupling the radial eigenmotions. The magnetron frequency is to first order constant while the mass-dependent reduced cyclotron frequency can be used to manipulate individual ion species. The technique's precision and accuracy

\footnotetext{
*Email: aniak@triumf.ca

${ }^{1}$ Current address: TRIUMF, 4004 Wesbrook Mall, Vancouver, BC V6T 2A3, Canada

${ }^{2}$ Current address: Department of Physics, Central Michigan University, Mount Pleasant, MI 48859, USA
}

rely on the preparation of low-emittance and pure ion bunches. Unfortunately, many ionization methods create not only the ion of interest but also contaminant ions. The problem of beam purity is exacerbated at radioactive isotope beam (RIB) facilities, where the radioactive ion of interest may be a small fraction of a cocktail beam and requires fast, efficient, and universal cleaning methods to minimize decay losses. Often, at mass spectrometers of radioactive isotopes, multi-step beam purification processes are employed (see e.g. $[3,4]$ and references therein). Unfortunately, most techniques such as mass-separating magnets lack the resolving power, $R=m / \Delta m \lesssim 5000$, necessary to remove isobaric or isomeric contaminants, $10^{3} \lesssim R \lesssim 10^{7}$.

Two high-resolution methods are currently employed Penning trap mass spectrometers: dipole cleaning $[1,5,6]$ and mass-selective buffer gas cooling $[7,8,9,10]$. Both rely on driving contaminant ions to a large radius such that they neither interact with the radioactive ion nor interfere with the measurement. The latter induces unstable magnetron motion, whose amplitude is increased by the dissipative force of the buffer gas; only the radioactive ion is centered by an excitation by an azimuthal quadrupolar RF-field at its mass-dependent cy- 
clotron frequency and extracted. The technique requires an additional Penning trap (to the measurement trap), adds beam preparation time of $\gtrsim 50$ $\mathrm{ms}$, and is incompatible for use with highly charged ions. In the former cleaning technique, each contaminant ion species is driven by a quick dipolar excitation at its mass-selective eigenfrequency, the identification of which repurposes limited RIB time from the mass measurement itself. The dipolar method can be modified to use time-separated dipolar excitations [11] (so-called Ramsey cleaning) resulting in higher resolving power, but it requires careful setup to avoid inadvertently exciting the radioactive ion with one of the many fringe peaks. The Stored Waveform Inverse Fourier Transform (SWIFT) [12] technique suggests itself as a compromise between the two approaches and has already been established for broadband excitations tailored to the needs of certain nuclear magnetic resonance and Fourier-transform ion-cyclotron-resonance experiments (see e.g. ref. [13] and references therein). SWIFT cleaning uses dipolar excitations to drive all ions within a user-defined mass band to large radii as opposed to dipole cleaning which acts on a single mass. Consequently, SWIFT does not require the exact identification of each contaminant ion.

The underlying principle of SWIFT is deceptively simple: design the desired excitation scheme in frequency space, compute its inverse Fourier transform to obtain the time-domain signal, and apply it to the ions in the Penning trap. For this work, the desired excitation, shown in figure 1 , is a notched waveform centered on the radioactive ion, with sufficient amplitude to drive undesired ions in the surrounding mass (or frequency) bands. To achieve a signal with a smaller dynamic range and with less distortion, a quadratic phase modulation in frequency space must be applied [14, 15]. To prevent power leakage which may inadvertently clean the radioactive ion, a window or apodization function may be multiplied to the waveform prior to the inverse Fourier transformation. An example of a SWIFT waveform in the time domain is shown in figure 1. At the Low Energy Beam and Ion Trap (LEBIT) facility [16], the SWIFT technique has been investigated for the removal of isobaric and high-lying isomeric contaminants. The technical challenges of its implementation are described, and first measurements, which demonstrate the great potential of the technique for high precision Penning trap mass spectrometers, are presented.

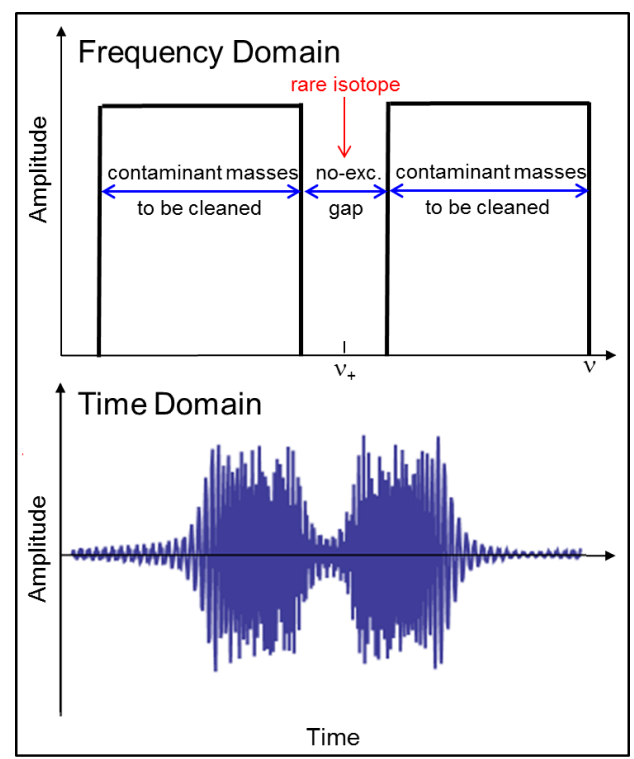

Figure 1: Excitation profiles for beam purification at LEBIT. (Top) In the frequency domain, two uniform rectangular excitations are separated by a gap of non-excitation. Ions whose $\nu_{+}$lies within the excitation bands are cleaned from the Penning trap. The $\nu_{+}$of the radioactive isotope is within the gap to prevent driving its reduced cyclotron motion. (Bottom) The real part of the inverse Fourier transform of the above signal, which has been phase modulated with a quadratic function and multiplied by an apodization function. 


\section{Experimental design}

\subsection{The LEBIT facility}

The LEBIT facility is located at the National Superconducting Cyclotron Laboratory (NSCL), where radioactive ion beams are produced by fast beam fragmentation and then filtered through the A1900 mass separator [17]. The relativistic fragments are slowed and thermalized with gas stopping techniques and then guided through a mass filter which selects ions by their charge-to-mass-number ratio (see [18] and references therein). The thermalization process often leads to molecular contamination due to space-charge and charge-exchange effects. When LEBIT operates off-line, stable beams are produced in a plasma-type ion source located just upstream of the beam cooler and buncher.

The beam cooler and buncher $[19,20]$ is a twostage linear RFQ trap filled with helium buffer gas. For collisional cooling, the first section is maintained at a high pressure, approximately $2 \times 10^{-2}$ mbar. In the final section of the device, the ions are accumulated and bunched in a low pressure $(\approx$ $10^{-4}$ mbar) environment. Neon gas may be substituted for or mixed with the helium buffer gas to increase the likelihood of collision-induced dissociation (CID) [4], in which a molecular ion is broken into a neutral and ionic part at masses smaller than the original molecules.

The approximately 100-ns ion bunches are sent to the Penning trap [21, 22]. A fast kicker operated as a time-of-flight (TOF) mass filter [4], resolving power $R \approx 300$, and a dynamic capture process into the Penning trap prevent trapping non-isobaric contaminants based on the TOF between the cooler and buncher and the Penning trap system. Therefore, all trapped ions have the same charge-to-massnumber ratio.

\subsection{The 9.4 T Penning trap system}

In the Penning trap, the magnetic field is provided by a $9.4 \mathrm{~T}$ superconducting solenoid magnet at LEBIT [21]. An electrostatic quadrupole potential is applied between the hyperbolic ring electrode and hyperbolic endcaps. The ion motion can be described by three eigenmotions [2, 23]. The axial motion is described by an oscillation about the trap center. The radial motion is a combination of the magnetron and the reduced cyclotron eigenmotions with eigenfrequencies $\nu_{-}$and $\nu_{+}$respectively. The former possess the lowest frequency, and to first order $\nu_{-}$is identical for all ions. The latter has the highest frequency and depends on the mass. Their sum equals the (true) cyclotron frequency [2], i.e.

$$
\nu_{+}+\nu_{-}=\nu_{c}
$$

The (true) cyclotron frequency is measured using the time-of-flight ion cyclotron resonance (TOFICR) detection technique [7, 24]. From the frequency ratio of a well known reference ion and the radioactive ion, the mass of the latter is easily deduced. Manipulation of the ions is achieved by applying azimuthal, multipole RF fields, for which the ring electrode is split eight-fold. Detailed descriptions of the LEBIT Penning trap can be found in $[21,22]$.

If the trapped ion sample contains impurities, the measured cyclotron frequency will be shifted [5] due to ion-ion interactions. For extremely high levels of contamination, the contaminant ions may completely obscure the signal from the radioactive ion species. Therefore, all contaminants must be removed prior to the mass measurements. Hitherto, the cleaning of isobaric contaminants at LEBIT has been performed with dipolar excitations at the reduced cyclotron frequency. The purification can be quick $\left(T_{\text {clean }} \lesssim 30 \mathrm{~ms}\right)$, requires little hardware or space, and has a demonstrated resolving power $>$ $10^{6}$ to separate isomers in ${ }^{70} \mathrm{Cu}^{+}$with $T_{\text {clean }}=3 \mathrm{~s}$ [6]. Unfortunately, the high resolving power costs efficiency as each contaminant ion must be identified. This identification process costs valuable RIB time otherwise spent on the mass measurement itself. Moreover, extremely low-level contaminants $(<5 \%)$ cannot be identified in a timely manner albeit they may shift the measured cyclotron frequency of the radioactive ion. Consequently, we desired a superior cleaning technique which satisfied the following criteria.

1. Provide fast cleaning, $T_{\text {clean }} \approx 30 \mathrm{~ms}$ to prevent losses due to radioactive decay

2. Be broadband to avoid the time-consuming identification of each contaminant ion

3. Excite a total bandwidth of about $30 \mathrm{kHz}$ with a non-excitation gap $g=1000 \mathrm{~Hz}$ centered at the reduced cyclotron frequency $\nu_{+}$of the radioactive ion to remove isobaric contaminants (E.g. at $A=30$ clean a mass range of of 0.2 atomic mass units)

4. Suppress the signal strength in the gap by a factor of 1000 compared to the amplitude of the excitation bands so as not to perturb the radioactive ion motion 


\subsection{SWIFT configuration}

Two configurations of SWIFT were investigated, which differed in the arbitrary waveform generator (AWG) chosen to generate the time-domain signal. The signals were investigated off-line with an Agilent EXA Signal Analyzer N9010A. Clearly a low noise floor in the AWG output is desirable. Calculations indicated that at least 14 -bit vertical resolution (the number of discrete levels in the voltage range) would be needed to satisfy criterion 3 above, sufficient noise suppression in the gap of the waveform. Another criterion for the AWG was the available bandwidth to cover the frequency range required at LEBIT. Typical reduced cyclotron frequencies range from $1.6 \mathrm{MHz}$ for $A / Q=85$ to 7.5 $\mathrm{MHz}$ for $A / Q=19$, where $A / Q$ is the ratio of the mass number to the charge state. For long cleaning times, e.g. $T_{\text {clean }}=100 \mathrm{~ms}$, large memory sizes $\mathrm{N} \geq 2$ MS (mega-samples) are required. Alternatively, the SWIFT waveform can be created over a smaller bandwidth, e.g. $50 \mathrm{kHz}$ (roughly $1 / 3$ of a mass unit at $\mathrm{A}=30$ ), and shifted to the frequency region of interest by amplitude modulation (AM) with a sine wave at a carrier frequency $\nu_{\text {carrier }}$. The modulation shifts the waveform from a frequency $\nu_{S W I F T}$ to $\nu_{S W I F T} \pm \nu_{\text {carrier. }}$. AM reduces not only the memory requirements of the AWG but also the time needed to upload the waveform into the AWG. The first instance of SWIFT at LEBIT implemented AM with the Tektronix 3102 AWG and quickly demonstrated the limitations of AM.

\subsection{SWIFT using amplitude modulation}

The Tektronix 3102 AWG features 14-bit resolution, $\mathrm{N}=101 \mathrm{kS}$ (kilo-sample) memory, a sample rate up to $250 \mathrm{MS} / \mathrm{s}$ for waveforms greater than 16 $\mathrm{kS}$, and two output channels. The first channel was used for the SWIFT waveform and the second for the carrier frequency. Two devices were tested for AM with qualitatively identical results: the AD835 "multiplier" chip and the Mini-Circuit ZAD-3H+ mixer.

The notched waveform directly from the Tektronix AWG satisfies the desired criteria; the modulated version, however, cannot ensure that the signal strength in the gap was suppressed by a factor of 1000 for an arbitrary $\nu_{+}$. The Tektronix AWG is linear to $-50 \mathrm{dBc}$ in the $\mathrm{MHz}$ regime. That is, the output of the AWG for the SWIFT signal $f_{S W I F T}$ is $\sum A_{i} f_{S W I F T}\left(i \nu_{0} t\right)$ and for the carrier sine wave is $\sum B_{j} \sin \left(j \nu_{\text {carrier }} t\right)$, where the amplitude $A_{i>1} \leq A_{1}-50 \mathrm{~dB}$ and similar for $B_{i}$. Not only do the first harmonics mix, but also all harmonics mix, described by $\sum_{i=1} \sum_{j=1} A_{i} B_{j}\left\{f_{S W I F T}\left[\left(i \nu_{0}+j \nu_{\text {carrier }}\right) t\right]+\right.$ $\left.f_{S W I F T}\left[\left(i \nu_{0}-j \nu_{\text {carrier }}\right) t\right]\right\}$. The result is a profusion of SWIFT signals distributed throughout the frequency domain. Figure 2 exemplifies the phenomenon for two carrier frequencies. The $i=1$, $j=1$ term is the fundamental and desired signal. All others are "cross terms." We are not concerned with the cross terms which fall entirely outside of the fundamental gap, centered at the radioactive ion's $\nu_{+}$. Those which do excite the radioactive ion and may cause systematic errors in the subsequent mass measurement. As a result of these cross terms, the choice of SWIFT waveform parameters and $\nu_{\text {carrier }}$ is non-trivial. Furthermore, AM devices are generally nonlinear and may introduce noise themselves, which also hinders the creation of the desired SWIFT signal. Consequently, we abandoned AM in favor of a large-memory AWG.

\subsection{SWIFT waveforms generated by large-N $A W G$}

The second AWG characterized for SWIFT was the Agilent 33521-02, which features $\mathrm{N}=16 \mathrm{MS}$ memory, 16-bit vertical resolution, and $250 \mathrm{MS} / \mathrm{s}$ output rate for waveforms larger than $16 \mathrm{kS}$. Its linearity in the $\mathrm{MHz}$ regime was $\approx-50 \mathrm{dBc}$, similar to other bench-top models. A test function and a sample SWIFT signal were used to explore the built-in filters [25] ("normal," "step," and none) for smoothing the transition between points in an arbitrary waveform. Without a filter, the output changes between points with $\mathrm{a} \approx 10$ ns transition time. The step filter was designed for step functions but suffers more roll-off relative than the normal filter. With its wide, flat frequency response, the normal filter was found to minimize noise and to have the most well defined gap. The off-line analysis with the spectrum analyzer indicated that SWIFT excitation schemes generated by the Agilent AWG satisfied all criteria.

\section{Results and discussion}

The SWIFT waveforms generated with the Agilent AWG were passed through a T\&C Power Conversion AG1021 RF amplifier (at 100\% gain) to one of the Penning trap ring electrode segments, creating to first order a dipolar field. Tests were first performed with ${ }^{23} \mathrm{Na}^{+}$and ${ }^{39} \mathrm{~K}^{+}$produced by 


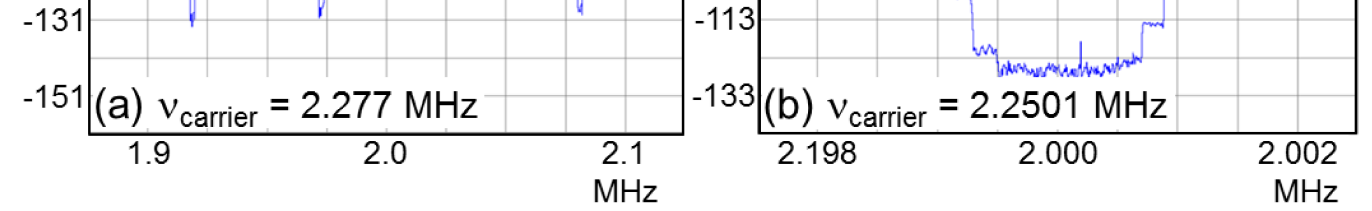

Figure 2: FFT of amplitude-modulated SWIFT signals obtained with the Agilent EXA Signal Analyzer N9010A. Each excitation band is $124 \mathrm{kHz}$ wide, and the gap is $2 \mathrm{kHz}$ wide. (a) Amplitude modulation at $\nu_{\text {carrier }}=2.277 \mathrm{MHz}$. (b) Amplitude modulation at $\nu_{\text {carrier }}=2.2501 \mathrm{MHz}$. The Tektronix AWG produces harmonics of the SWIFT and modulating sine signals. As all terms mix, "cross terms" appear in addition to the fundamental SWIFT signal, the largest waveform in (a) and (b). The parameters were chosen to illustrate the effect of cross terms. Depending on the choice of $\nu_{0}$ and $\nu_{\text {carrier }}$, the cross terms can project outward from the fundamental SWIFT signal (a) or into the fundamental signal's gap (b). The latter excites the radioactive ion and may introduce systematic errors in the subsequent mass measurement.

heating the tungsten filament of the off-line test ion source. To find the required cleaning amplitude for a given $T_{\text {clean }}$ and bandwidth, a single excitation band was generated and centered on the reduced cyclotron frequency of ${ }^{23} \mathrm{Na}^{+}$, and the amplitude was scanned. A linear fit was made to the slope of the count distribution of ions ejected from the Penning trap and detected on a micro-channel plate; the value at zero counts was taken as the cleaning amplitude. As expected, the cleaning amplitude increases with the bandwidth and inversely with the cleaning time. Typically an amplitude just above the minimum was used to reduce power broadening which could narrow the gap.

The apodization window was decided by scanning the edge of the excitation band over the reduced cyclotron frequency of ${ }^{23} \mathrm{Na}^{+}$, ejecting the remaining ions, and detecting them on a detector downstream of the Penning trap. Three cases were tested empirically in addition to no apodization. First, a suggestion given in [14]

$$
w_{k}= \begin{cases}+\sin (2 \pi k / N) & \text { if } 0 \leq k \leq \frac{N}{8} \\ 1 & \text { if } \frac{N}{8} \leq k \leq \frac{7 N}{8} \\ -\sin (2 \pi k / N) & \text { if } \frac{7 N}{8} \leq k \leq N\end{cases}
$$

and referred to here as the "quarter-wave sine" was used. Second, the Hanning window, common to digital signal processing, was investigated.

$$
w_{k}=\sin ^{2}\left(\frac{\pi k}{N}\right) \text { for } 0 \leq k<N
$$

Third, the Connes window is defined by

$$
w_{k}=\left(1-\frac{(k-N / 2)^{2}}{(N / 2)^{2}}\right) \text { for } 0 \leq k<N .
$$

All cases, shown in Figure 3, exhibit "wiggles" in the count distribution (indicating wiggles in the power distribution) due to discontinuities in the waveform. While the amplitude of the excitation band is reduced at the edge, the signal apodized by the Hanning window features the smallest side lobes. As it is more important to suppress stray

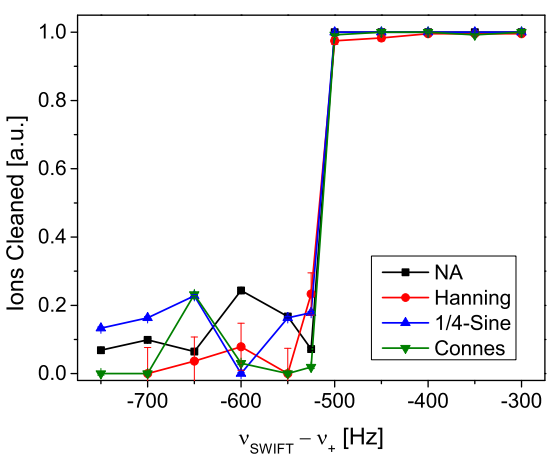

Figure 3: Recorded ion signal as a function of frequency for different apodization windows. The edge of a rectangular excitation band was scanned over $\nu_{+}$of ${ }^{23} \mathrm{Na}^{+}$. The Hanning wave function was chosen to minimize power leakage into the gap. (Parameters are listed in table 1 except here only one band of $1 \mathrm{kHz}$ was used.)

power inside the gap than outside, the Hanning window was chosen.

\begin{tabular}{lccc}
\hline Parameter & Symbol & \multicolumn{2}{c}{ Value } \\
\hline Cleaning time & $T_{\text {clean }}$ & 20 & $\mathrm{~ms}$ \\
Excitation band, each & $b$ & 1000 & $\mathrm{~Hz}$ \\
Gap bandwidth & $g$ & $1000 \mathrm{~Hz}$ \\
Cleaning amplitude & & \multicolumn{2}{c}{$180 \mathrm{~V}_{p p}$} \\
Apodization function & & \multicolumn{2}{c}{$\mathrm{Hanning}^{23} \mathrm{Na}^{+}$} \\
Ion species & & \multicolumn{2}{c}{}
\end{tabular}

Table 1: Typical parameter values used to generate the excitation profiles depicted in figures $3,5,6$, and 7 . The gap was centered at the reduced cyclotron frequency $\nu_{+}\left({ }^{23} \mathrm{Na}^{+}\right)$ $=6254817 \mathrm{~Hz}$. Any difference between table and figures is given in the figure captions.

After the minimum cleaning amplitude had been established and the apodization function chosen, the SWIFT profile was scanned by shifting the center frequency over the reduced cyclotron frequency of the trapped ion and counting the remaining ions on the downstream detector. Figure 4 compares the expected SWIFT excitation scheme to the count distribution of ions cleaned from the Penning trap. The measured excitation profiles closely match ex- 


\begin{tabular}{clccccc}
\hline Sub-figure & Ion & $\mathrm{T}_{\text {clean }}[\mathrm{ms}]$ & $\mathrm{b}[\mathrm{Hz}]$ & $\mathrm{g}[\mathrm{Hz}]$ & Amp. $\left[\mathrm{V}_{p p}\right]$ & Apodization \\
\hline $\mathrm{a}, \mathrm{b}$ & ${ }^{39} \mathrm{~K}^{+}$ & 20 & 500 & 100 & 95 & Hanning \\
$\mathrm{c}, \mathrm{d}$ & ${ }^{39} \mathrm{~K}^{+}$ & 20 & 500 & 300 & 95 & Hanning \\
$\mathrm{e}, \mathrm{f}$ & ${ }^{23} \mathrm{Na}^{+}$ & 20 & 1000 & 500 & 180 & Hanning \\
\hline
\end{tabular}

Table 2: Parameter values used to generate the excitation profiles depicted in figure 4

pectations. The observed profiles are more square than calculations as the cleaning amplitude used was slightly higher than the minimum needed to clean.

\subsection{Maximum resolving power}

To determine the smallest gap width $g$, which in turn determines the resolving power, $R \approx$ $\nu_{+} /(g / 2)$, we used the same sequence of steps as for a mass measurement, but we used SWIFT cleaning instead of dipolar cleaning and only applied the quadrupolar excitation at $\nu_{c}$. The gap was centered on $\nu_{+}$and its width varied. Two quantities were examined: the number of ions and the time of flight of the ions between trap and detector. Three cases could be identified. If the amplitude at $\nu_{+}$(i.e. a wide gap) was zero, no cleaning occurred; the number of ions and the TOF were the same as that measured without SWIFT. If the amplitude was greater than needed to clean (e.g. for $g=0$ ), then no ions were observed. If the amplitude was in between these two extremes, then not all ions were cleaned and the TOF was reduced. Measurements were performed for $T_{\text {clean }}=10,20$, and $50 \mathrm{~ms}$ with a fixed excitation bandwidth as described in table 1 . The results were the same when expressed as the product of the gap width and $T_{\text {clean }}$. A conservative estimate of the minimum gap, at which no excitation of the ion of interest occurs, is $6 / T_{\text {clean }}$, which corresponds to $120 \mathrm{~Hz}, 300 \mathrm{~Hz}$, and $600 \mathrm{~Hz}$ for $T_{\text {clean }}$ $=50,20,10 \mathrm{~ms}$ respectively. Figure 5 shows the count distribution and the mean time of flight for $T_{\text {clean }}=20 \mathrm{~ms}$. High resolution scans of the gap itself indicate that residual power leaks into the gap, particularly at the edges, despite the apodization and the 16-bit amplitude resolution. The leakage may result in non-coherent dipolar excitation of the ion of interest, whose effect was investigated with a high precision mass measurement.

\subsection{Effect on accuracy of mass measurement}

To perform a mass measurement, the cyclotron frequency of the radioactive ion is measured using the TOF-ICR method $[24,7]$ and compared
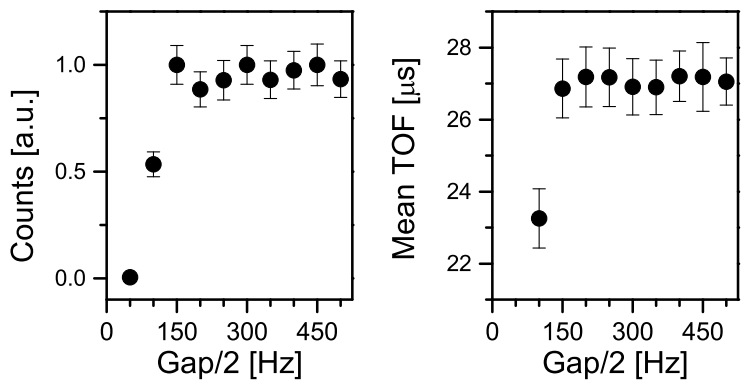

Figure 5: (a) Detected ${ }^{23} \mathrm{Na}^{+}$ions as the gap size was varied from 0 to $500 \mathrm{~Hz}$. (b) Mean TOF of ${ }^{23} \mathrm{Na}^{+}$ions as a function of gap width. The minimum gap for $T_{\text {clean }}=20 \mathrm{~ms}$ is 300 $\mathrm{Hz}$. When these measurements were performed with other cleaning times, it was found that the smallest gap is given by $6 / T_{\text {clean }}$. (The parameter values used to generate the SWIFT waveform can be found in table 1.)

to that of a well known reference ion to calibrate the magnetic field. Thus, the primary experimental result is the ratio $R=\nu_{c, 1} / \nu_{c, 2}=m_{2} / m_{1}$ if the ions have the same charge state. To study the effect of SWIFT under typical working conditions, we measured the cyclotron frequency of ${ }^{39} \mathrm{~K}^{+}$with and without SWIFT on pure ion samples. The SWIFT parameters can be found in table 3 , and the quadrupolar excitation was a single $\pi$-pulse applied over $500 \mathrm{~ms}$. The TOF resonances obtained

\begin{tabular}{lccc}
\hline Parameter & Symbol & \multicolumn{2}{c}{ Value } \\
\hline Cleaning time & $T_{\text {clean }}$ & 20 & $\mathrm{~ms}$ \\
Excitation band, each & $b$ & 5000 & $\mathrm{~Hz}$ \\
Gap bandwidth & $g$ & $1000 \mathrm{~Hz}$ \\
Cleaning amplitude & & 182 & $\mathrm{~V}_{p p}$ \\
Apodization function & & \multicolumn{2}{c}{ Hanning } \\
Gap center & & \multicolumn{2}{c}{$\nu_{+}\left({ }^{39} \mathrm{~K}^{+}\right)$} \\
\hline
\end{tabular}

Table 3: SWIFT parameter values used to determine the effect of SWIFT cleaning on the accuracy of a mass measurement of ${ }^{39} \mathrm{~K}^{+}$, (see figure 6). The cyclotron frequency of ${ }^{39} \mathrm{~K}^{+}$was measured with and without SWIFT cleaning prior to the quadrupolar excitation. Here $\nu_{+}\left({ }^{39} \mathrm{~K}^{+}\right)=3$ $689441 \mathrm{~Hz}$.

with and without SWIFT cleaning were consistent with one another. The measured ratios are plotted in figure 6 . The mean ratio was found to be consis- 


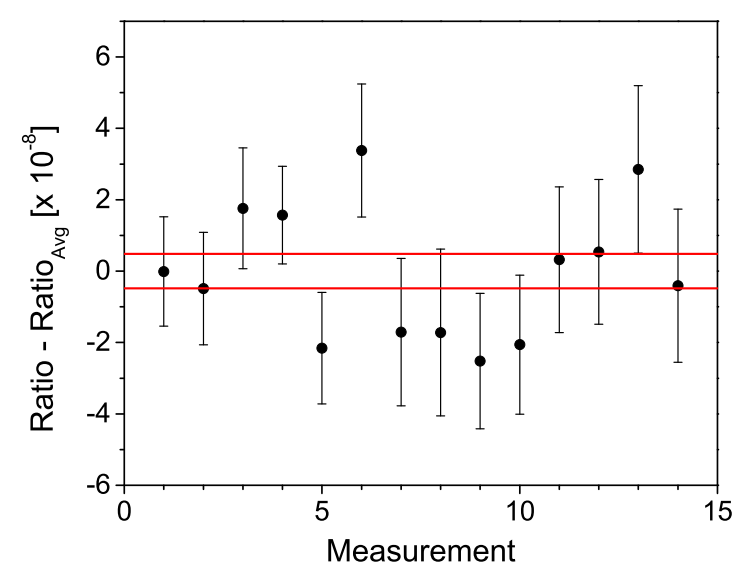

Figure 6: Deviations from the mean value of the mass measurement of ${ }^{39} \mathrm{~K}^{+}$with SWIFT-based cleaning. Calibrations were performed without cleaning. Measurements were performed with SWIFT cleaning. (SWIFT waveform parameters are listed in table 1.) The mean ratio is 1.000000002 $7(48)$; and, the Birge ratio is 1.02(13).

tent with unity, $1.0000000027(48)$, or a relative deviation of $2.7(4.8) \times 10^{-9}$; the uncertainty corresponds to $176 \mathrm{eV}$ in the mass of ${ }^{39} \mathrm{~K}$. The Birge ratio is $1.02(13)$ [26], which indicates no additional systematic uncertainty. Hence, the deployment of SWIFT under these conditions does not affect the accuracy of high precision Penning trap mass spectrometry.

\subsection{Isobaric separation}

The ultimate test for SWIFT was performed under typical working conditions: simultaneously trap two isobaric species, clean one, and measure the cyclotron frequency of the other. To this end, a mixture of carbon monoxide $(\mathrm{CO})$ and nitrogen $\left(\mathrm{N}_{2}\right)$ gases were ionized in the plasma-type ion source and delivered to the Penning trap. The ratio of $\mathrm{CO}^{+}$to $\mathrm{N}_{2}^{+}$in the Penning trap was approximately 2. Their separation is $2061 \mathrm{~Hz}$ and requires $R 2.5$ $\times 10^{3}$. This represents a typical measurement at LEBIT, where the radioactive ion's $\nu_{+}$is usually a few $\mathrm{kHz}$ or more from the contaminants'. First a resonance was taken with both species in the trap with an excitation time of $7 \mathrm{~ms}$ and a $2700 \mathrm{~Hz}$ range; a resonance was observed for each species, see figure 7a. Second, a SWIFT signal was generated with a $1 \mathrm{kHz}$ gap and $5 \mathrm{kHz}$ bands $\left(T_{\text {clean }}\right.$ $=20 \mathrm{~ms}$ and $180 \mathrm{~V}_{p p}$ ). In figure $7 \mathrm{~b}$, the gap was centered on the reduced cyclotron frequency of $\mathrm{N}_{2}^{+}$; because SWIFT cleaned the $\mathrm{CO}^{+}$from the Penning

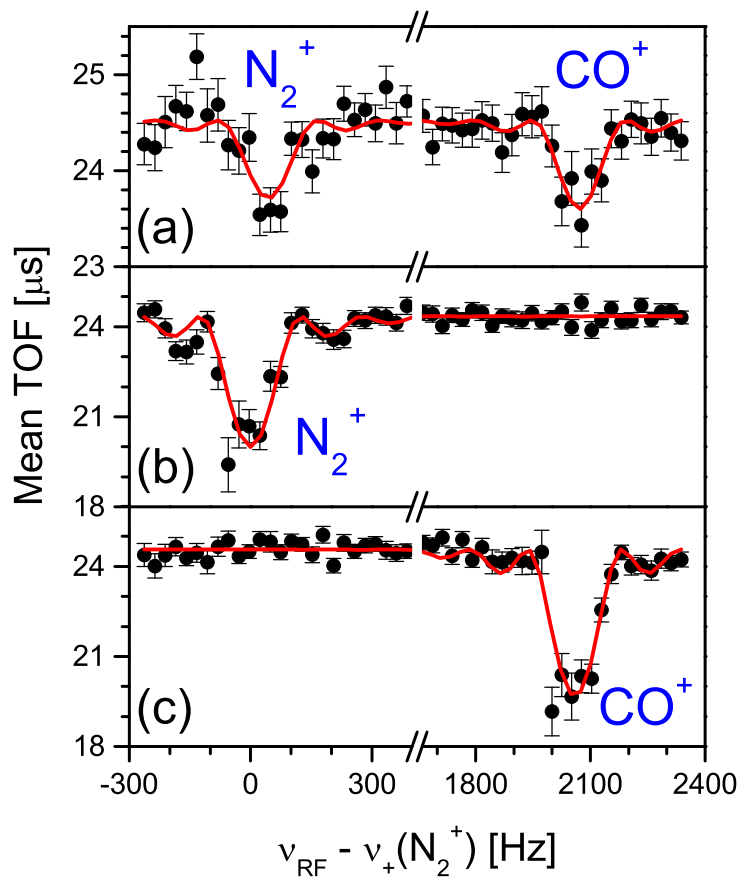

Figure 7: Quadrupole resonances $\left(\mathrm{T}_{R F}=7 \mathrm{~ms}\right)$ with $\mathrm{CO}^{+}$ and $\mathrm{N}_{2}^{+}$simultaneously loaded in the Penning trap. (a) SWIFT off. A small resonance is visible for each species. (b) SWIFT cleaning $\mathrm{CO}^{+}$. Only the $\mathrm{N}_{2}^{+}$resonance is observed. (c) SWIFT cleaning $\mathrm{N}_{2}^{+}$. Only the $\mathrm{CO}^{+}$resonance is seen. The red curves are theoretical line shapes [23] to data. The resonances were measured over 3.5 hours, during which time the decay in the magnetic field caused a shift of 7 $\mathrm{Hz}$ in the measured $\nu_{c}$ of $\mathrm{CO}^{+}$. Note that the vertical scale in (a) differs from that in (b) and (c). (SWIFT waveform parameters are as listed in table 2 with $b=5 \mathrm{kHz}$ here.) 
trap, only the resonance of $\mathrm{N}_{2}^{+}$was observed. Similarly, when the gap was centered on $\nu_{+}$of $\mathrm{CO}^{+}$ for figure $7 \mathrm{c}$, only the $\mathrm{CO}^{+}$resonance was observed as SWIFT cleaned the $\mathrm{N}_{2}^{+}$. The simultaneous presence of the contaminant ions (figure 7a) reduces the signal strength compared to when only one species is present (figures $7 \mathrm{~b}$ and c). Not only do the latter exhibit deeper resonances, but also their sidebands are more pronounced.

\section{Summary and final remarks}

SWIFT has been implemented for the first time for high precision Penning trap mass spectrometry of radioactive isotopes. Its success relied on high amplitude resolution to lower the noise floor, a large AWG memory to avoid the need for amplitude modulation, and sufficient amplification to drive out contaminant ions. All of the criteria for success outlined in section 2 have been met or exceeded. The minimum useable gap was found to be $6 / T_{\text {clean }}$ or $600 \mathrm{~Hz}$ at $T_{\text {clean }}=10 \mathrm{~ms}$, the shortest cleaning times envisioned at LEBIT. The resolving power then can be approximated by $R=$ $\nu_{+} T_{\text {clean }} / 3$. For the case of ${ }^{23} \mathrm{Na}^{+}, R$ ranges from $4.2 \times 10^{4}$ for the typical LEBIT $T_{\text {clean }}=20 \mathrm{~ms}(\Delta \nu$ $=300 \mathrm{~Hz})$ to $2.1 \times 10^{5}$ for $T_{\text {clean }}=100 \mathrm{~ms}(\Delta \nu=$ $60 \mathrm{~Hz}$ ). Successful isobaric separation was demonstrated with the $\mathrm{CO}-\mathrm{N}_{2}$ mass doublet. It follows, then, that certain isomeric states (of order $500 \mathrm{keV}$ or higher) will also be expelled from the trap. It should be noted that the resolving power will be reduced for higher masses since $\nu_{+}$roughly scales with the magnetic field strength and inversely with the mass. Nonetheless, resolving powers of $\sim 10^{4}$ can easily be obtained for $A=100$, even for $T_{\text {clean }}<$ $50 \mathrm{~ms}$.

In comparison to other popular isobaric separation methods, SWIFT offers several advantages. The traditional dipole cleaning at LEBIT requires the exact identification of each contaminant species. SWIFT eliminates this time-consuming process and allows more beam time to be devoted to the measurement of radioactive isotopes. Mass-selective buffer gas cooling, developed at ISOLTRAP [7, 8], is slow in comparison to dipole or SWIFT cleaning. Recently, another interesting approach has been proposed, SImultaneous Magnetron and resonant COnversion (SIMCO) $[27,28]$. While the initial investigations [27] shows promise, SIMCO has yet to be tested under realistic conditions (i.e. isobar separation) and possible effects on accuracy still need to be checked. At present, SWIFT cleaning offers the best compromise: fast $\left(T_{\text {clean }}=20 \mathrm{~ms}\right)$ and efficient (no contaminant identification) cleaning with the high resolution needed for isobaric purification $\left(R>10^{4}\right)$ and with no effect on the precision or accuracy of the mass measurement.

\section{Acknowledgements}

A.A.K. would like to thank D. Morris for his assistance and many fruitful discussions. This work was supported by Michigan State University, the National Science Foundation under Contract No. PHY-0606007, and the DOE under Contract No. DE-FG02-00ER41144.

\section{References}

[1] K. Blaum, High-accuracy mass spectrometry with stored ions, Physics Reports 425 (1) (2006) 1-78. doi:10.1016/j.physrep.2005.10.011.

[2] L. Brown, G. Gabrielse, Precision spectroscopy of a charged particle in an imperfect Penning trap, Physical Review A 25 (4) (1982) 2423-2425. doi:10.1103/PhysRevA.25.2423.

[3] Y. Blumenfeld, T. Nilsson, P. Van Duppen, Facilities and methods for radioactive ion beam production, Phys. Scripta T152 (2013) 014023. doi:10.1088/00318949/2013/T152/014023.

[4] P. Schury, G. Bollen, M. Block, D. J. Morrissey, R. Ringle, A. Prinke, J. Savory, S. Schwarz, T. Sun, Beam purification techniques for low energy rare isotope beams from a gas cell, Hyperfine Interactions 173 (1-3) (2006) 165-170. doi:10.1007/s10751-007-9553-0.

[5] G. Bollen, S. Becker, H.-J. Kluge, M. Knig, R. Moore, T. Otto, H. Raimbault-Hartmann, G. Savard, L. Schweikhard, H. Stolzenberg, ISOLTRAP: a tandem Penning trap system for accurate on-line mass determination of short-lived isotopes, Nuclear Instruments and Methods in Physics Research Section A: Accelerators, Spectrometers, Detectors and Associated Equipment 368 (3) (1996) 675-697. doi:10.1016/01689002(95)00561-7.

[6] K. Blaum, D. Beck, G. Bollen, P. Delahaye, C. Guénaut, F. Herfurth, A. Kellerbauer, H.-J. Kluge, U. Köster, D. Lunney, S. Schwarz, L. Schweikhard, C. Yazidjian, Laser ionization and Penning trap mass spectrometry a fruitful combination for isomer separation and high-precision mass measurements, Hyperfine Interactions 162 (1-4) (2006) 173-179. doi:10.1007/s10751-005-9223-z.

[7] G. Bollen, R. B. Moore, G. Savard, H. Stolzenberg, The accuracy of heavy-ion mass measurements using time of flight-ion cyclotron resonance in a Penning trap, Journal of Applied Physics 68 (9) (1990) 4355. doi:10.1063/1.346185.

[8] G. Savard, S. Becker, G. Bollen, H.-J. Kluge, R. Moore, T. Otto, L. Schweikhard, H. Stolzenberg, U. Wiess, A new cooling technique for heavy ions in a Penning trap, Physics Letters A 158 (5) (1991) 247-252. doi:10.1016/0375-9601(91)91008-2. 
[9] H. Raimbault-Hartmann, D. Beck, G. Bollen, M. König, H.-J. Kluge, E. Schark, J. Stein, S. Schwarz, J. Szerypo, A cylindrical Penning trap for capture, mass selective cooling, and bunching of radioactive ion beams, Nuclear Instruments and Methods in Physics Research Section B: Beam Interactions with Materials and Atoms 126 (14) (1997) 378-382. doi:10.1016/S0168-583X(96)01067-

[10] V. S. Kolhinen, S. Kopecky, T. Eronen, et al., JYFLTRAP: a cylindrical Penning trap for isobaric beam purification at IGISOL, Nucl. Instrum. Meth. A 528 (2004) 776

[11] T. Eronen, V.-V. Elomaa, U. Hager, J. Hakala, A. Jokinen, A. Kankainen, S. Rahaman, J. Rissanen, C. Weber, J. yst, Preparing isomerically pure beams of shortlived nuclei at JYFLTRAP, Nuclear Instruments and Methods in Physics Research Section B: Beam Interactions with Materials and Atoms 266 (19-20) (2008) 4527-4531. doi:10.1016/j.nimb.2008.05.076.

[12] A. Marshall, T. Wang, T. Ricca, Tailored excitation for Fourier transform ion cyclotron mass spectrometry, Journal of the American Chemical Society 107 (26) (1985) 7893-7897. doi:10.1021/ja00312a015.

[13] S. Guan, A. Marshall, Stored waveform inverse Fourier transform (SWIFT) ion excitation in trapped-ion mass spectometry: Theory and applications, International Journal of Mass Spectrometry and Ion Processes 157158 (1996) 5-37. doi:10.1016/S0168-1176(96)04461-8.

[14] L. Chen, T. Wang, T. Ricca, A. Marshall, Phasemodulated stored waveform inverse Fourier transform excitation for trapped ion mass spectrometry, Analytical Chemistry 59 (3) (1987) 449-454. doi:10.1021/ac00130a016.

[15] S. Guan, General phase modulation method for stored waveform inverse Fourier transform excitation for Fourier transform ion cyclotron resonance mass spectrometry, The Journal of Chemical Physics 91 (2) (1989) 775. doi:10.1063/1.457129

[16] R. Ringle, P. Schury, T. Sun, G. Bollen, D. Davies, J. Huikari, E. Kwan, D. Morrissey, A. Prinke, J. Savory, S. Schwarz, C. Sumithrarachchi, Precision mass measurements with LEBIT at MSU, International Journal of Mass Spectrometry 251 (2-3) (2006) 300-306. doi:10.1016/j.ijms.2006.02.011.

[17] D. Morrissey, B. Sherrill, M. Steiner, A. Stolz, I. Wiedenhoever, Commissioning the A1900 projectile fragment separator, Nuclear Instruments and Methods in Physics Research Section B: Beam Interactions with Materials and Atoms 204 (2003) 90-96. doi:10.1016/S0168-583X(02)01895-5.

[18] G. Bollen, C. Campbell, S. Chouhan, C. Gunaut, D. Lawton, F. Marti, D. Morrissey, J. Ottarson, G. Pang, S. Schwarz, A. Zeller, P. Zavodszky, Manipulation of rare isotope beams from high to low energies, Nuclear Instruments and Methods in Physics Research Section B: Beam Interactions with Materials and Atoms 266 (19-20) (2008) 4442-4448. doi:10.1016/j.nimb.2008.05.058.

[19] S. Schwarz, G. Bollen, D. Lawton, A. Neudert, R. Ringle, P. Schury, T. Sun, A second-generation ion beam buncher and cooler, Nuclear Instruments and Methods in Physics Research Section B: Beam Interactions with Materials and Atoms 204 (2003) 474-477. doi:10.1016/S0168-583X(02)02114-6.

[20] T. Sun, S. Schwarz, G. Bollen, D. Lawton, R. Ringle,
P. Schury, Commissioning of the ion beam buncher and cooler for LEBIT, The European Physical Journal A 25 (S1) (2005) 61-62. doi:10.1140/epjad/i2005-06-1269.

[21] R. Ringle, G. Bollen, D. Lawton, P. Schury, S. Schwarz, T. Sun, The LEBIT 9.4 T Penning trap system, The European Physical Journal A 25 (S1) (2005) 59-60. doi:10.1140/epjad/i2005-06-132-y.

[22] R. Ringle, G. Bollen, A. Prinke, J. Savory, P. Schury, S. Schwarz, T. Sun, The LEBIT 9.4 T Penning trap mass spectrometer, Nuclear Instruments and Methods in Physics Research Section A: Accelerators, Spectrometers, Detectors and Associated Equipment 604 (3) (2009) 536-547. doi:10.1016/j.nima.2009.03.207.

[23] M. König, G. Bollen, H.-J. Kluge, T. Otto, J. Szerypo, Quadrupole excitation of stored ion motion at the true cyclotron frequency, International Journal of Mass Spectrometry and Ion Processes 142 (1-2) (1995) 95-116. doi:10.1016/0168-1176(95)04146-C.

[24] G. Gräff, H. Kalinowsky, J. Traut, A direct determination of the proton electron mass ratio, Zeitschrift für Physik A: Atoms and Nuclei 297 (1) (1980) 35-39. doi:10.1007/BF01414243.

[25] Agilent 33500 series $30 \mathrm{MHz}$ function / arbitrary waveform generator user's guides.

[26] R. Birge, The calculation of errors by the method of least squares, Physical Review 40 (2) (1932) 207-227. doi:10.1103/PhysRev.40.207.

[27] M. Rosenbusch, K. Blaum, C. Borgmann, S. Kreim, M. Kretzschmar, D. Lunney, L. Schweikhard, F. Wienholtz, R. Wolf, Buffer-gas-free mass-selective ion centering in Penning traps by simultaneous dipolar excitation of magnetron motion and quadrupolar excitation for interconversion between magnetron and cyclotron motion, International Journal of Mass Spectrometry 325327 (2012) 51. doi:10.1016/j.ijms.2012.06.008.

[28] M. Kretzschmar, Theory of simultaneous dipole and quadrupole excitation of the ion motion in a Penning trap, International Journal of Mass Spectrometry 325327 (2012) 30-44. doi:10.1016/j.ijms.2012.07.015. 

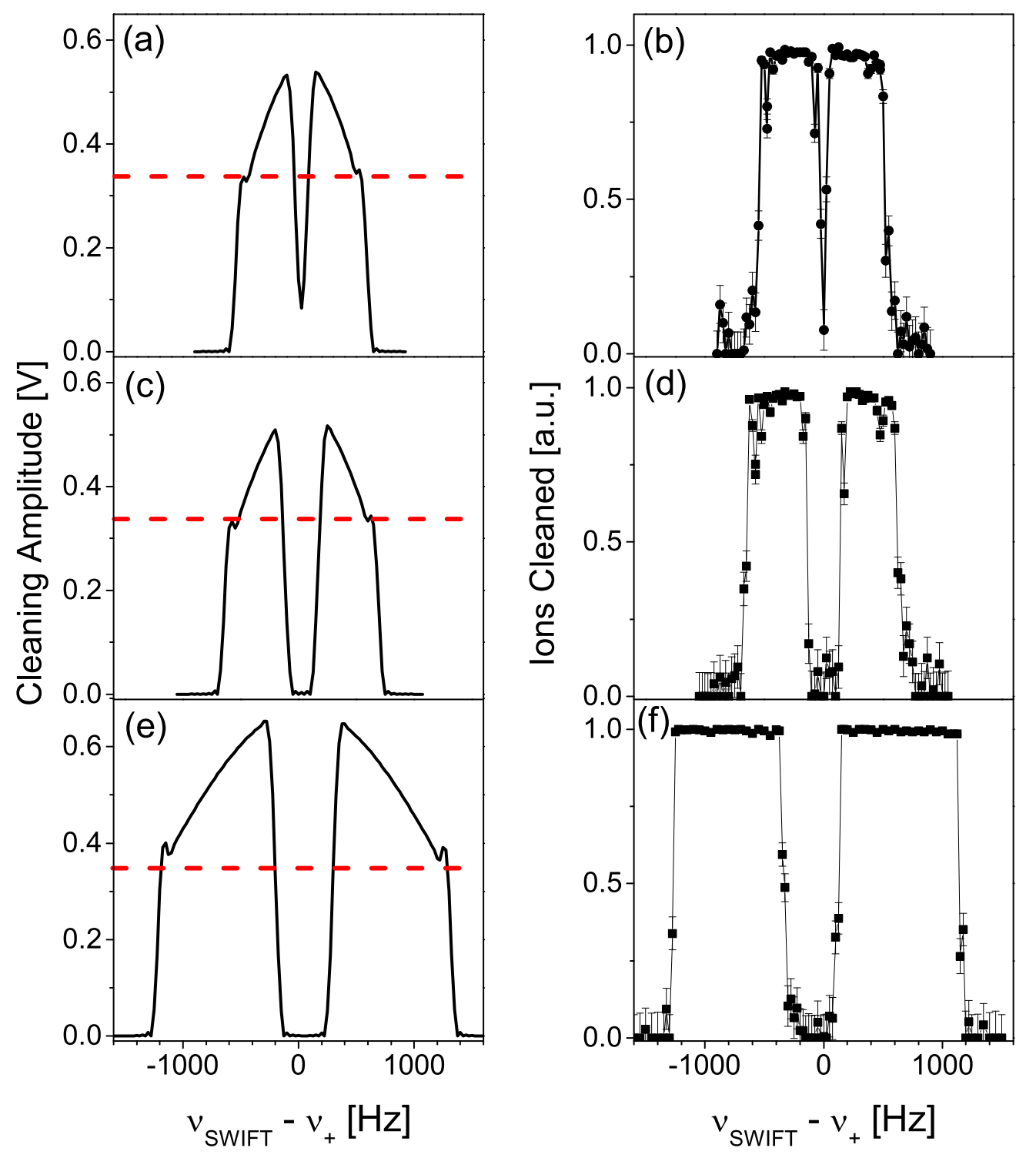

Figure 4: Comparison of expected SWIFT excitation profiles and observed ion profiles. The red dashed line indicates the amplitude required to remove ions. Left: The magnitude of the forward Fourier transform of the SWIFT signal. Right: Ions cleaned defined as (1 - fraction of remaining ions) as the SWIFT signal was shifted over $\nu_{+}$in frequency space. The gap size was set as follows $(\mathrm{a}, \mathrm{b}) \mathrm{g}=100 \mathrm{~Hz},(\mathrm{c}, \mathrm{d}) \mathrm{g}=300 \mathrm{~Hz}$, and $(\mathrm{e}, \mathrm{f}) \mathrm{g}=500 \mathrm{~Hz}$. (b,d) were experimentally determined with ${ }^{39} \mathrm{~K}^{+}$ and (f) was measured using ${ }^{23} \mathrm{Na}^{+}$. (The other parameter values to generate the SWIFT waveforms can be found in table 2.) 